\title{
Drug Accountability Sequence Number
}

National Cancer Institute

\section{Source}

National Cancer Institute. Drug Accountability Sequence Number. NCI Thesaurus. Code C87877.

A number that identifies the ordering relations (in time, space, etc.) in a set of drug accountability data. 